\title{
MINIMUM QUANTITY LUBRICANT FLOW ANALYSIS IN END MILLING PROCESSES: A COMPUTATIONAL FLUID DYNAMICS APPROACH
}

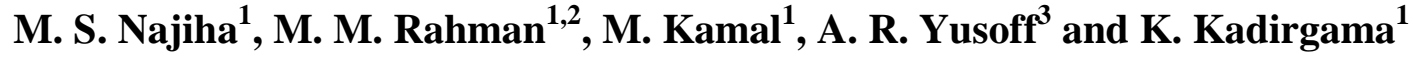 \\ ${ }^{1}$ Faculty of Mechanical Engineering \\ Universiti Malaysia Pahang, 26600 Pekan, Pahang, Malaysia \\ Phone: +6094242246; Fax: +6094242202 \\ E-mail: mustafizur@ump.edu.my \\ ${ }^{2}$ Automotive Engineering Centre \\ Universiti Malaysia Pahang \\ 26600 Pekan, Pahang, Malaysia \\ ${ }^{3}$ Faculty of Manufacturing Engineering \\ Universiti Malaysia Pahang, 26600 Pekan, Pahang, Malaysia
}

\begin{abstract}
This paper presents a two-dimensional steady-state incompressible analysis for the minimum quantity of lubricant flow in milling operations using a computational fluid dynamics (CFD) approach. The analysis of flow and heat transfer in a four-teeth milling cutter operation was undertaken. The domain of the rotating cutter along with the spray nozzle is defined. Operating cutting and boundary conditions are taken from the literature. A steady-state, pressure-based, planar analysis was performed with a viscous, realizable $\mathrm{k}-\varepsilon$ model. A mixture of oils and air were sprayed on the tool, which is considered to be rotating and is at a temperature near the melting temperature of the workpiece. Flow fields are obtained from the study. The vector plot of the flow field shows that the flow is not evenly distributed over the cutter surface, as well as the uneven distribution of the lubricant in the direction of the cutter rotation. It can be seen that the cutting fluid has not completely penetrated the tool edges. The turbulence created by the cutter rotation in the proximity of the tool throws oil drops out of the cutting zone. The nozzle position in relation to the feed direction is very important in order to obtain the optimum effect of the MQL flow.
\end{abstract}

Keywords: Minimum quantity lubricant, end milling, nozzle viscous flow, steady state, turbulence.

\section{INTRODUCTION}

Minimum Quantity Lubrication (MQL) is a sustainable manufacturing technique that is safe for the environment, the worker and is cost effective (Marksberrya \& Jawahir, 2008; Shen, 1996). The minimization of metal working fluids is a direct indicator of sustainable manufacturing. The goal of MQL is to machine parts using the minimal amount of metal working fluids, so that the workpiece, chips and environment remain dry after cutting (Boubekri \& Shaikh, 2012; Kumar \& Singh, 2010; Yusoff, 2011). Automotive, aerospace and other major manufacturing industrial segments are heavily involved in milling operations to produce components. A considerable amount of heat is generated during milling processes. This heat is mainly due to the high plastic deformation in the primary shear zone, and to the friction of the chip on the tool rake face. These conditions of friction and temperature cause tool wear, resulting in a poor surface finish and inexact dimensions. Cutting fluids are used to reduce the detrimental 
effects of heat and friction on both the tool and workpiece. The cutting fluid produces three positive effects in the process: heat elimination, lubrication at the chip-tool interface and chip removal (Khan, Rahman, Kadirgama, Maleque, \& Ishak, 2011; Singh 2010).

Metal working fluids or cutting fluids are used to minimize the thermal expansion of the worked metals, and thus aid in achieving a better surface finish on the finished product and a longer tool life (Khan et al., 2011; Rupinder \& Bhupinder, 2011). Keeping these benefits in mind, cutting fluids constitute a very important part from the very start of the manufacturing process. Depending on the workpiece, production structure and production location, the costs related to cooling lubricants range from 7\% to $17 \%$ of the total cost of the manufactured workpiece (Weinert, Inasaki, Sutherland, \& Wakabayashi, 2004). Due to growing environmental awareness and stringent regulations by governments, strict consideration is being given to the role of cutting fluids. Furthermore, the costs associated with the maintenance, service, disposal and waste management of cutting fluids have led to the search for new methods and production strategies with a minimum, or ideally no amount, of cutting fluids employed. Operator safety and health issues are also important concerns. Regulatory constraints, environmental protection, general public awareness and the need for cost-reduction have all promoted the development of new environmentally-conscious machining processes. The main problem in replacing or eliminating cutting fluids is that the energy generated in the machining process and dissipated as heat causes elevated temperatures, thermal damage, and dimensional inaccuracies. Cutting fluids are a vital factor in controlling these undesirable effects, mainly by providing lubrication and cooling. Lubrication reduces machining power and associated heat generation, while also enhancing surface quality and reducing tool wear. The cooling by the fluid removes heat from the tool and the workpiece. An ideal replacement or alternative to conventional flooded cooling and lubricating practice is dry machining, without the use of any cutting fluid. Research on dry machining was mainly concerned with the development of appropriate tools and coatings (Klocke \& Eisenblatter, 1997; Kobayashi, Ohgoe, Ozeki, Sato, Sumiya, Hirakuri, \& Aoki, 2005; Machado \& Wallbank, 1997; Nouari, List, Girot, \& Coupard, 2003; Reddy \& Rao, 2006; Su, Liu, Su, Yao, Kao, \& Cheng, 2006). Although dry machining is possible in some situations, there are still many issues regarding lubricity, tool life, thermal damage to the workpiece and others (Davim, Sreejith, \& Silva, 2007; Itoigawa, Childs, Nakamura, \& Belluco, 2006; Sun, Wong, Rahman, Wang, Neo, Tan, \& Onozuka, 2006). Therefore the MQL was proposed. The use of MQL is of great significance as a concession between substantial cutting fluid application and dry machining. It can reduce the quantity of heat generated due to friction and provide some cooling over the tool-workpiece interface and hence keep the workpiece temperatures lower than those in completely dry machining. Another characteristic of this technology is that when properly applied, both the parts and chips remain dry and are easier to handle. Previously conducted research has shown the advantages of MQL as opposed to the use of a conventional emulsion coolant, and there are several successful experiences following its use in the machining of different materials. However, in the field of high-speed milling two important factors remain to be studied; the correct penetration of the MQL jet into the cutting zone and the relative position between the nozzle and tool. The present study aims to investigate the spraying of an oil-compressed air mixture onto the cutting zone, with one nozzle placed horizontally to the tool feed direction using the CFD method. 


\section{METHODS AND MATERIALS}

A four-teeth milling cutter is modeled for the study, rotating at $2000 \mathrm{rpm}$. A compressed air and oil mixture is assumed as the cutting media. Mixture properties are shown in Table 1. The mass fraction of the oil mist is assumed to be constant throughout the compressed air. ANSYS Fluent is used for the analysis. A steady-state, pressure-based, planar analysis is conducted. A viscous, realizable $\mathrm{k}-\varepsilon$ model is used. A pressurevelocity coupled scheme is used with a 200 courant number. The nozzle employed is 2 $\mathrm{mm}$ in diameter, and is placed at $0^{\circ}$ (horizontal) to the tool's axis of rotation. The density of the air-oil mixture is taken as the weighted average of the air and oil densities. An unstructured mesh with trigonal elements is used, with a maximum mesh size of $0.004 \mathrm{~mm}$; the grid is shown in Figure 1. The mesh is divided into two regions, with the region around the tool being densely meshed to more accurately predict the flow field in a high turbulence region.

Table 1. Mixture Properties

\begin{tabular}{ll}
\hline \multicolumn{1}{c}{ Properties } & \multicolumn{1}{c}{ Value } \\
\hline Compressed air pressure $(\mathrm{MPa})$ & 0.4 \\
Air viscosity $(\mathrm{kg} / \mathrm{m}-\mathrm{s})$ & $1.7894 \times 10^{-5}$ \\
Air density $\left(\mathrm{kg} / \mathrm{m}^{3}\right)$ & 1.225 \\
Air volume flow rate $(1 / \mathrm{min})$ & 72.6 \\
Compressed air inlet flow velocity $(\mathrm{m} / \mathrm{s})$ & 231 \\
Oil viscosity $\left(\mathrm{kg} / \mathrm{m}^{-\mathrm{s})}\right.$ & $88 \times 10^{-4}$ \\
Density of oil $\left(\mathrm{kg} / \mathrm{m}^{3}\right)$ & $0.96 \times 10^{3}$ \\
Specific heat of oil $(\mathrm{J} / \mathrm{kg})$ & 2.0 \\
Thermal conductivity of oil $(\mathrm{W} / \mathrm{Mk})$ & 231 \\
Oil volume flow rate $(\mathrm{ml} / \mathrm{hr})$ & 15 \\
\hline
\end{tabular}

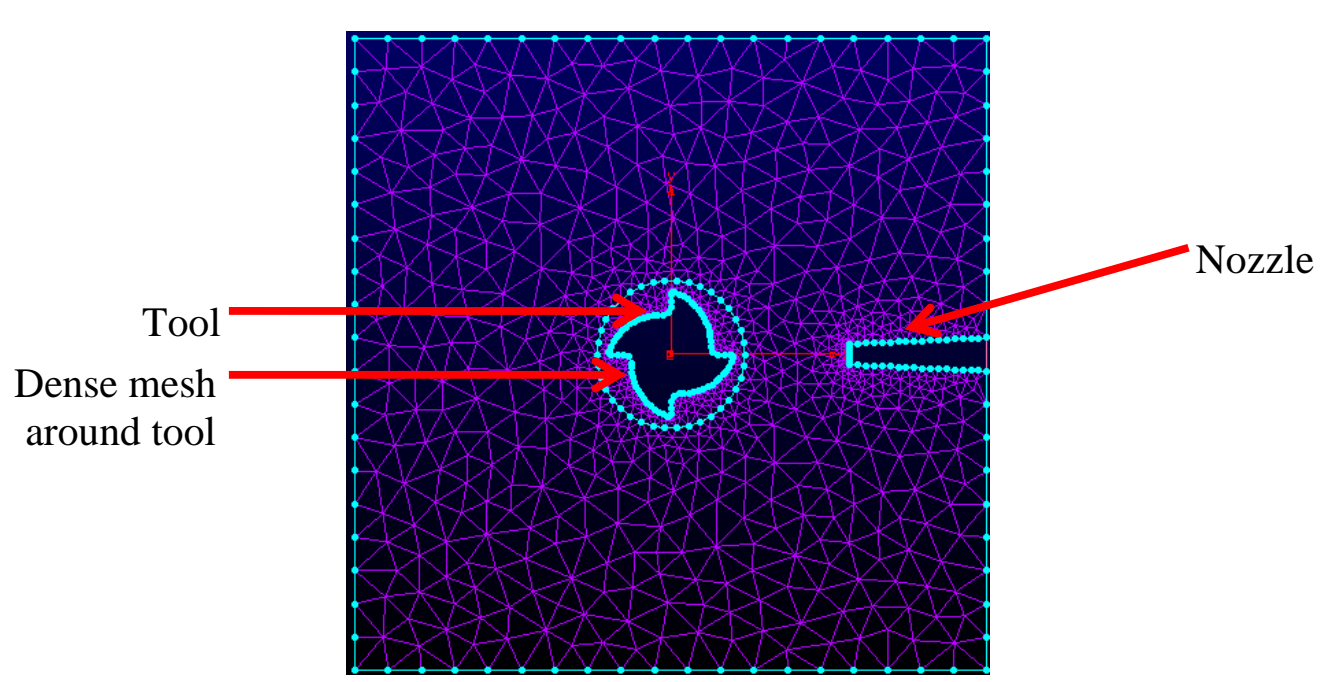

Figure 1. Meshing grid of the tool and nozzle.

\section{RESULTS AND DISCUSSION}

The injection of a MQL flow on a rotating end mill is simulated in this study. Figure 2 shows the velocity field (vector plot) for the tool and the MQL jet. Velocity contours are 
shown in Figure 3. The turbulence close to the tool means the nozzle flow is unable to penetrate into the cutting zone. This may result from the fact that the mass flow is lower than that necessary for penetration. Close to the tool, a centrifugal effect is generated which obstructs the path of the coolant towards the cutting zone.

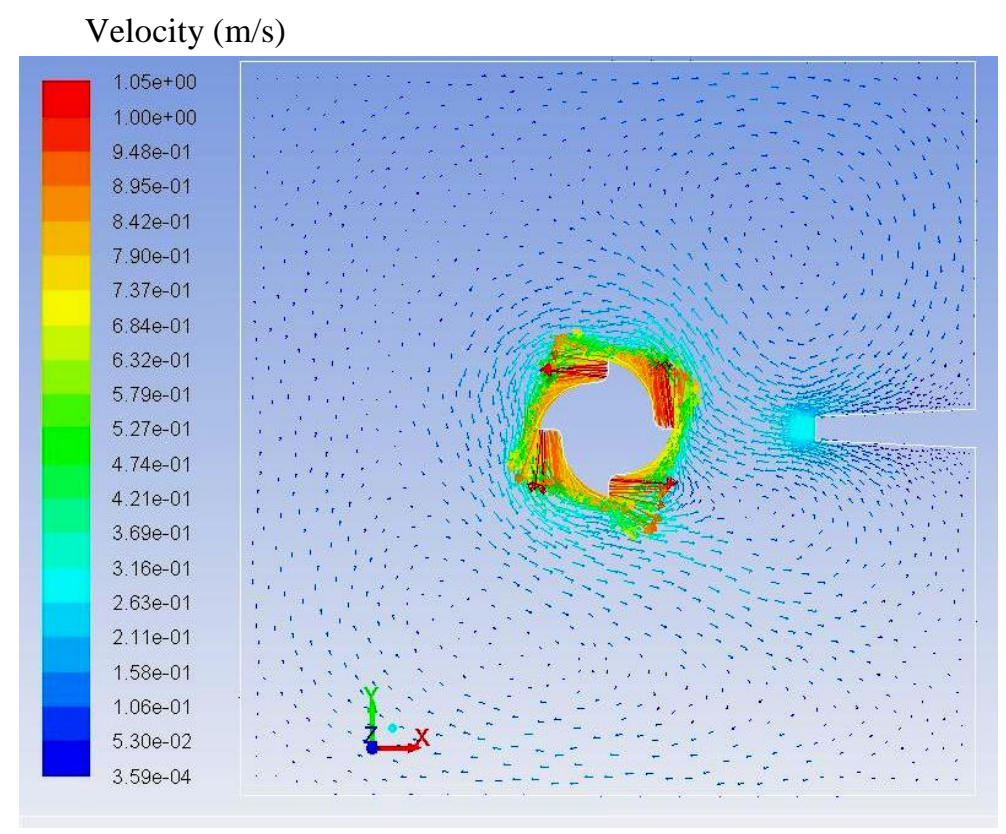

Figure 2. Velocity field (vector plot) for the tool and MQL jet.

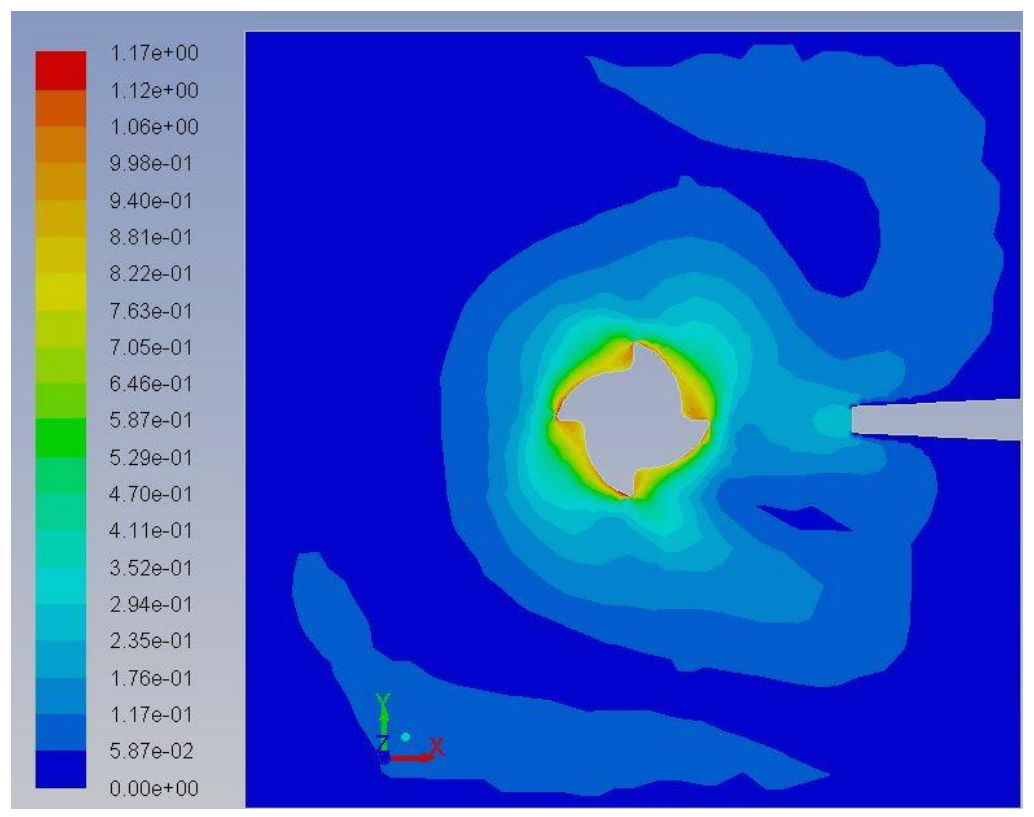

Figure 3. Velocity contours of the MQL

Path lines for the jet are shown in Figure 4. The vector plot of the flow field shows that the flow is not evenly distributed, or not evenly lubricating the cutter surface. The cutting fluid does not completely penetrate to the tool edges. The vortex or the turbulence originated by cutter rotation in the proximity of the tool throws the oil drops out of the cutting zone, i.e. pushing the lubricant jet away from the cutter surface. 
Furthermore, the MQL jet flow is very small. The mass flow rate can be increased to force the flow through the vortex. The issue of uneven distribution can be solved by increasing the number of nozzles, thus distributing the incoming lubricant evenly on the cutter's periphery which can result in the improved lubrication of the cutting surface. Velocity contours also show the uneven distribution of the lubricant in the direction of the cutter rotation. The nozzle position in relation to the feed direction is very important in order to obtain the optimum effect from the MQL flow.

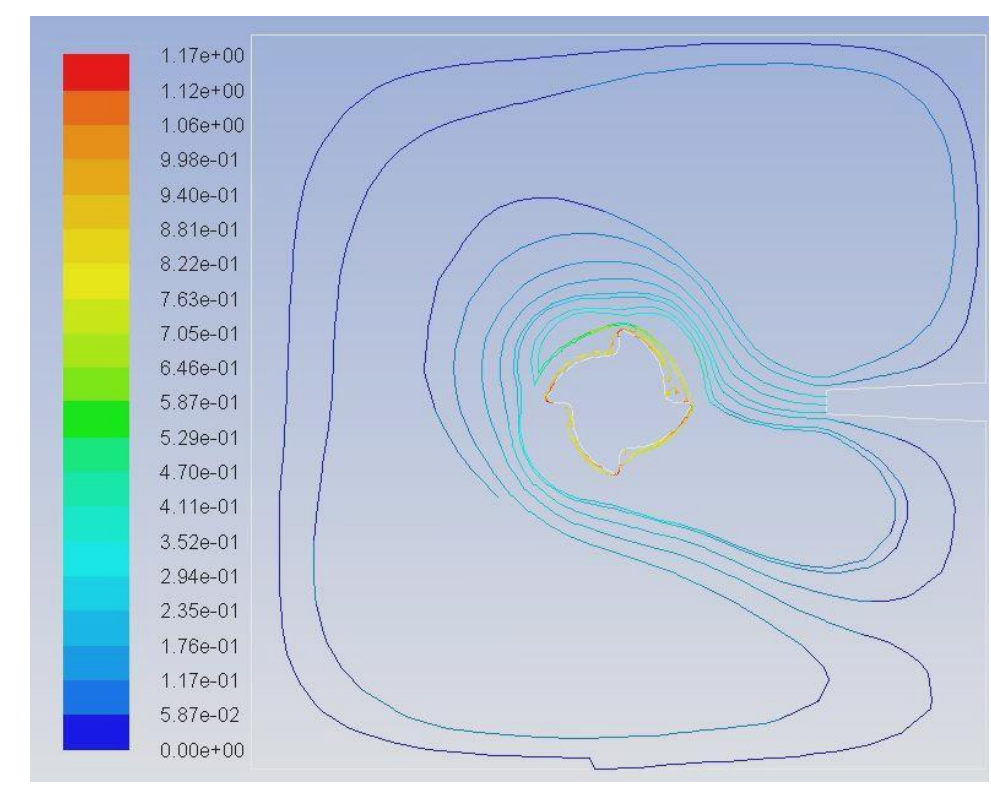

Figure 4. Pathlines for the MQL jet

\section{CONCLUSIONS}

The CFD analysis shows that the flow penetration into the cutting zone is dependent upon the flow velocity and the number of nozzles. A single nozzle with a very small flow velocity cannot achieve complete lubrication using MQL fluid. The MQL flow in the present study is inefficient in high-speed milling, because it is not able to reach the inner zones of the tool teeth. In reality several nozzles, placed according to the feed direction, are necessary. A 3-D comprehensive analysis should be conducted for the detailed analysis of MQL in end milling operations. An analysis using more than one nozzle should also be carried out.

\section{ACKNOWLEDGMENTS}

The authors would like to thank the Universiti Malaysia Pahang for financial support under Grant No. RDU110110.

\section{REFERENCES}

Boubekri, N., \& Shaikh, V. (2012). Machining using minimum quantity lubrication: a technology for sustainability. International Journal of Applied Science and Technology, 2 (1), 111-115 
Davim, J. P., Sreejith, P. S., \& Silva, J. (2007). Turning of brasses using minimum quantity of lubricant and flooded lubricant conditions. Materials and Manufacturing Processes, 22, 45-50.

Itoigawa, F., Childs, T. H. C., Nakamura, T., \& Belluco, W. (2006). Effects and mechanisms in minimal quantity lubrication machining of aluminum alloy. Wear, 260 (3), 339-344.

Khan, M. A. R., Rahman, M. M., Kadirgama, K., Maleque, M. A., \& Ishak, M. (2011). Prediction of surface roughness of Ti-6Al-4V in electrical discharge machining: a regression model. Journal of Mechanical Engineering and Sciences, 1, 16-24.

Klocke, F., \& Eisenblatter, G. (1997). Dry cutting. Annals of the CIRP, 46, 519-526.

Kobayashi, S., Ohgoe, Y., Ozeki, K., Sato, K., Sumiya, T., Hirakuri, K. K., \& Aoki, H. (2005). Diamond-like carbon coatings on orthodontic archwire. Diamond and Related Materials, 14, 1094-1097.

Kumar, S., \& Singh, R. (2010). Investigation of surface properties in manganese powder mixed electrical discharge machining of OHNS and D2 die steels. International Journal of Automotive and Mechanical Engineering. 2, 181-199.

Machado, A. R., \& Wallbank, J. (1997). Effect of extremely low lubricant volumes in machining. Wear, 210, 76-82.

Marksberrya, P. W., \& Jawahir, I. S. (2008). A comprehensive tool-wear/tool-life performance model in the evaluation of NDM (near dry machining) for sustainable manufacturing. International Journal of Machine Tools and Manufacture, 48, 878-886.

Nouari, M., List, G., Girot, F., \& Coupard, D. (2003). Experimental analysis and optimisation of tool wear in dry machining of aluminium alloys. Wear, 255, 1359-1368.

Reddy, N. S. K., \& Rao, P. V. (2006) .Selection of an optimal parametric combination for achieving better surface finish in dry milling using genetic algorithms. International Journal of Advanced Manufacturing Technology, 28, 463-473.

Rupinder, S., \& Bhupinder S. (2011). Comparison of cryo-treatment effect on machining characteristics of titanium in electric discharge machining. International Journal of Automotive and Mechanical Engineering, 3, 239-248.

Shen, C. H. (1996). The importance of diamond coated tools for agile manufacturing and dry machining. Surface and Coating Technology, 86-87, 672-677.

Singh, R. (2010). Comparison of statistically controlled machining solutions of titanium alloys using USM. International Journal of Automotive and Mechanical Engineering, 1, 66-78.

Su, Y. L., Liu, T. H., Su, C. T., Yao, S. H., Kao, W. H., \& Cheng, K. W. (2006). Wear of $\mathrm{CrC}$-coated carbide tools in dry machining. Journal of Materials Processing Technology, 171, 108-117.

Sun, J., Wong, Y. S., Rahman, M., Wang, Z. G., Neo, K. S., Tan, C. H., \& Onozuka, H. (2006). Effects of coolant supply methods and cutting conditions on tool life in end milling titanium alloy. Machining Science and Technology, 10, 355-370.

Yusoff, A. R. (2011). Literature review of optimization technique for chatter suppression in machining. Journal of Mechanical Engineering and Sciences, 1, 47-61.

Weinert, K., Inasaki, I., Sutherland, J. W., \& Wakabayashi, T. (2004). Dry machining and minimum quantity lubrication. CIRP Annals - Manufacturing Technology, $53(2), 511-537$. 